\title{
Genómica del síndrome de Down
}

Díaz-Cuéllar S1, Yokoyama-Rebollar E², Del Castillo-Ruiz V³

\section{Resumen}

El síndrome de Down es la cromosomopatía más común del ser humano, con una frecuencia de 1 en 650 recién nacidos vivos. Las manifestaciones clínicas son muy variables y dependen, en gran parte, de la presencia de diversos factores genéticos como mosaicismo, cambios variables en el número de copias o variantes de un solo nucleótido. La identificación de estas variantes se ha convertido en un tema central de investigación ya que es esencial para la comprensión de los mecanismos moleculares subyacentes en esta enfermedad.

PALABRAS CLAVE: síndrome de Down, trisomía 21, Hsa21, genómica.

Acta Pediatr Mex. 2016 Sep;37(5):289-296.

\section{Genomics of Down syndrome.}

Díaz-Cuéllar S ${ }^{1}$, Yokoyama-Rebollar E², Del Castillo-Ruiz V³

\section{Abstract}

Down syndrome is the most common chromosomal abnormality in humans with a frequency of 1 in 650 live births. Clinical manifestations are highly variable and depend largely on the presence of various genetic factors as mosaicism, copy number variants changes or single nucleotide variants. The identification of these variants has become a central topic of research, since it is essential for understanding the molecular mechanisms underlying this disease.

KEYWORDS: Down syndrome; trisomy 21; Hsa 21; genomics
${ }^{1}$ Médico residente de tercer año. Departamento de Genética Humana.

${ }^{2}$ Doctora en ciencias médicas. Médico adscrito del departamento de Genética Humana.

${ }^{3}$ Especialista en genética médica. Jefa del Departamento de Genética Humana.

Instituto Nacional de Pediatría, México.

Recibido: 27 de mayo del 2016

Aceptado: 13 de junio del 2016

\section{Correspondencia}

Dra. Emiy Yokoyama-Rebollar

eyr75@hotmail.com.

Este artículo debe citarse como

Díaz-Cuéllar S, Yokoyama-Rebollar E, Del CastilloRuiz V. Genómica del síndrome de Down. Acta Pediatr Mex. 2016;37(5):289-296. 


\section{INTRODUCCIÓN}

El síndrome de Down es la alteración cromosómica más frecuente y la causa principal de discapacidad intelectual en todo el mundo. En la mayoría de los casos su causa es una copia extra del cromosoma 21 (human chromosome 21 - Hsa21). Abarca un conjunto complejo de patologías que involucran prácticamente todos los órganos y sistemas. Las alteraciones más prevalentes y distintivas son la dificultad para el aprendizaje, dismorfias craneofaciales, hipotiroidismo, cardiopatías congénitas, alteraciones gastrointestinales y leucemias. Se estima que es la causa de 1 de cada 150 abortos del primer trimestre y de $8 \%$ de las anomalías congénitas en Europa. ${ }^{1,2}$

Fue descrito por John Langdon Down en 1866, dentro de su propuesta de clasificación de pacientes con discapacidad intelectual. ${ }^{3}$ Se asoció por primera vez con una alteración cromosómica en 1959, cuando Lejeune, Gautier y Turpin describieron 5 niños y 4 niñas con discapacidad intelectual y 47 cromosomas en el cultivo de fibroblastos, siendo un acrocéntrico pequeño el cromosoma extra. Los autores propusieron que el origen de este cromosoma extra se debía probablemente a una falta de disyunción, que por lo tanto ésta era la razón por la que la frecuencia del padecimiento aumentaba con la edad materna. ${ }^{4}$

El síndrome de Down se debe a una trisomía completa Hsa21 o una trisomía parcial que incluye la región crítica 21q22.3. El 95\% de los casos se debe a una trisomía completa o regular; alrededor de 3\% se debe a mosaicismo, una alteración en la que los pacientes tienen conjuntamente células normales y células con un Hsa21 extra; menos de $2 \%$ se origina por una traslocación no equilibrada; es decir, un cariotipo con 46 cromosomas, pero uno de ellos, usualmente el cromosoma 14, contiene material cromosómico extra del Hsa21.
La Organización Mundial de la Salud estima una prevalencia mundial de 1 en cada 1,000 recién nacidos vivos; sin embargo, estas cifras varían, lo que refleja que la prevalencia depende de variantes socioculturales, como el acceso al diagnóstico prenatal y la interrupción legal del embarazo. ${ }^{5}$ En México, la Secretaría de Salud estima una prevalencia de 1 en 650 recién nacidos vivos; 6 pero el informe de 2010 del Registro y Vigilancia Epidemiológica de Malformaciones Congénitas Externas (RYVEMCE) estimó una tasa de 14.32 por 10,000 recién nacidos vivos: 1 en $698 .^{7}$

El diagnóstico es clínico y se confirma por citogenética. El patrón de características físicas observables (Gestalt) es altamente sugestivo, así como las alteraciones sistémicas. Sin embargo, no todas las alteraciones están presentes en cada individuo afectado. En recién nacidos el diagnóstico puede dificultarse; no obstante, diez características son altamente prevalentes. Hall, en 1966, analizó 48 recién nacidos afectados y encontró que $100 \%$ tuvieron 4 o más características y $89 \%$ tuvieron 6 o más. Desde entonces, estas características se utilizan para evaluar a todo recién nacido vivo, conocidas como criterios de Hall ${ }^{8}$ (Cuadro 1).

Cuadro 1. Criterios de Hall

\begin{tabular}{|l|l|}
\hline Característica & $\%$ \\
\hline Perfil facial plano & 90 \\
\hline Reflejo de moro disminuido & 85 \\
\hline Hipotonía & 80 \\
\hline Hiperlaxitud & 80 \\
\hline Piel redundante en nuca & 80 \\
\hline Fisuras palpebrales oblicuas hacia arriba & 80 \\
\hline Displasia de cadera & 70 \\
\hline Clinodactilia del quinto dedo & 60 \\
\hline Pabellones auriculares displásicos & 60 \\
\hline Pliegue palmar transverso & 45 \\
\hline
\end{tabular}

Hall B. Clin Pediatr.1966;5(1):4-12. 
En la década de los 50 del siglo pasado sólo $47 \%$ de los recién nacidos vivos con síndrome de Down sobrevivían un año; esta cifra se incrementó a más de 90\% en los 80 y de 1983 a 1997 la esperanza de vida aumentó de 25 a 49 años. Los factores de riesgo que influyen en la sobrevida son: madre de raza negra, cardiopatía congénita, defectos mayores no cardiacos y prematurez. Los predictores de sobrevida no difieren en pacientes con síndrome de Down comparados con pacientes con discapacidad intelectual en general. ${ }^{9}$

La morbilidad de los pacientes con síndrome de Down implica costos médicos 12 a 13 veces mayores en comparación con la población general durante los primeros cuatro años de vida, especialmente los pacientes con cardiopatía congénita que tienen la mayor mortalidad y en quienes se estima que requieren de 5 a 7 veces más atención médica que los pacientes con síndrome de Down sin cardiopatía congénita. ${ }^{10}$ Otras causas frecuentes de hospitalización son complicaciones de leucemia, respiratorias, hipotiroidismo y demencia; las respiratorias causan incluso mayor mortalidad. ${ }^{11}$

El antecedente de un hijo con translocación de novo no representa un riesgo incrementado y la recurrencia tiene que relación solamente con la edad materna. Sin embargo, si el padre es portador de una translocación robertsoniana el riesgo es de 3 a 5\%; si la madre es la portadora el riesgo aumenta 10 a 15\%. La situación es diferente cuando alguno de los padres es portador de una translocación 21:21, ya que el riesgo de recurrencia es de $100 \%$.

\section{MANIFESTACIONES CLÍNICAS Y SU ASO- CIACIÓN CON LA GENÓMICA}

Aunque algunas características de los pacientes con síndrome de Down son muy constantes existe gran variabilidad fenotípica. Diversos estudios en ratones y humanos han intentado identificar genes sensibles a dosis que expliquen de forma individual cada uno de los datos clínicos. Inicialmente se delimitó una región del Hsa21 llamada región crítica del síndrome de Down; sin embargo, otros estudios han determinado diferentes regionesque también contribuyen al fenotipo. $^{12}$

\section{Manifestaciones neurológicas}

\section{Desarrollo}

Los pacientes adquieren los hitos del desarrollo de forma tardía tanto en el área motora como en el lenguaje. El coeficiente intelectual promedio en pacientes con síndrome de Down es de 35 a 70 puntos. ${ }^{13}$ Estudios en ratones han sugerido que los defectos en la neurogénesis, transmisión sináptica y vías de señalización celular podrían contribuir al problema del desarrollo a través de una inhibición excesiva de la neurotransmisión. ${ }^{14}$ Estudios en individuos con trisomía parcial de Hsa21 han sugerido diversas regiones del Hsa21 que contribuyen con esta discapacidad intelectual; ${ }^{15}$ sin embargo, estudios en ratones no confirmaron estos hallazgos. ${ }^{16}$

Existen diversos genes en la región crítica del síndrome de Down. El gen DYRK1A (21q22.13) que se expresa en el sistema nervioso en desarrollo y del adulto, su función es la inhibición de la proliferación celular y promoción de la diferenciación neuronal prematura. Estudios en ratón que sobreexpresa Dyrk1a, mostraron problemas de aprendizaje graves, así como defectos de memoria espacial. ${ }^{17}$ De igual forma, el gen SIM2 (21q22.13) ortólogo al gen Drosophila single minded, es un factor de transcripción y principal regulador del desarrollo; también se expresa en el cerebro humano en desarrollo y en ratones transgénicos que sobreexpresan $\operatorname{Sim} 2$, han demostrado problemas de aprendizaje leve y problemas de memoria. ${ }^{18}$ La molécula de adhe- 
sión del síndrome de Down (DSCAM, 21q22.2) se expresa en dendritas neuronales y contribuye a la plasticidad sináptica; sin embargo, inhibe la ramificación de las dendritas cuando se sobreexpresa en las neuronas del hipocampo in vitro y en el ratón con tres copias de Dscam..$^{19}$ Otro gen asociado con la discapacidad intelectual es el Kcnj6 (GIRK2, 21q22.1), el cual se ha visto sobreexpresado en el hipocampo en ratón. ${ }^{20}$

También existen genes fuera de la región crítica del síndrome de Down que se han asociado al fenotipo neurológico de los pacientes con síndrome de Down. La synaptojanin1 (SYNJ1, 21q22.2) es una proteína, formadora de vesículas en la sinapsis neuronal, que juega un papel importante en la neurotransmisión desfosforilando el fosfatidilinositol bifosfato alterado en un modelo ratón con síndrome de Down que tenía problemas de aprendizaje y de memoria, que se normalizaron al reducir la dosis génica de Synj 1 de tres a dos. ${ }^{21}$ Finalmente, el análisis de trisomías segmentarias confirma el papel importante de la proteína precursora amiloide (APP, 21q21.3) ya que los inhibidores de los metabolitos de esta proteína, en un modelo ratón, mejoraron su aprendizaje y memoria, sugiriendo que la triple dosis del gen APP podría ser causante del fenotipo neurológico en pacientes con síndrome de Down. ${ }^{22}$

\section{Control motor e hipotonía}

Los neonatos con síndrome de Down comúnmente presentan hipotonía y la mayoría alteraciones motoras. Los hallazgos en humanos y en modelos ratón han mostrado un número reducido de neuronas granulares en el cerebelo. ${ }^{23}$ Esta neurogénesis cerebelar reducida podría deberse a un defecto de la señalización de sonic hedgehog $(\mathrm{SHH})$ en neuronas precursoras, causado por niveles elevados de APP. ${ }^{24}$ Otro gen ya mencionado es el DYRK $1 A$, que también se propone como gen candidato para el déficit motor en estos pacientes.
Otra teoría son los defectos en la morfología de la sinapsis y en la formación de vesículas sinápticas en la unión neuromuscular. Fortaleciendo esta teoría, la sobreexpresión de los genes ITSN1 (21q22.1), SYNJ1 (21q22.2) y DSCR1 (21q22.12) en moscas transgénicas, homólogos en Drosophila, causaron defectos locomotores y falla en el reciclaje de las vesículas en la unión neuromuscular, sugiriendo que estos tres genes, junto con DYRK $1 A$ y APP, son genes candidato dosis-sensibles causantes de los defectos motores en pacientes con síndrome de Down. ${ }^{25}$

\section{Enfermedad de Alzheimer}

Los pacientes con síndrome de Down se caracterizan por presentar Alzheimer a edades tempranas y el eventual inicio de demencia. Un gen candidato importante es el ya mencionado $A P P$, ya que su proteólisis genera amiloide $\beta$ $(A-\beta)$, el principal componente de las placas de amiloides en cerebros de pacientes con enfermedad de Alzheimer y cuyas mutaciones de tipo duplicaciones se han relacionado con inicio temprano de dicha enfermedad. ${ }^{26}$ Otro gen implicado, también ya mencionado, es el DYRK1A, cuyo producto ha mostrado que fosforila a la proteína APP. Un modelo ratón que sobreexpresa Dyrk1a muestra niveles elevados de fosfo-APP y A- $\beta .{ }^{27}$

\section{Características craneofaciales}

Microcefalia, occipital plano, braquicefalia, cara pequeña y maxilares de tamaño reducido son características del síndrome de Down. Estudios en modelos ratón han revelado un patrón craneofacial de anormalidades similares a éstas, e incluso se delimitó una región responsable de dichas características. ${ }^{28}$ Esta región contiene el gen Ets2 (ETS2, 21q22.2) cuya sobreexpresión mostró una asociación con las alteraciones esqueléticas observadas en pacientes con síndrome de Down. ${ }^{29}$ 


\section{Alteraciones hemato-oncológicas}

\section{Hematológicas}

Los pacientes con síndrome de Down tienen mayor riesgo de padecer leucemia (riesgo relativo de 18) y de forma particular la leucemia megacarioblástica tiene un riesgo relativo mucho mayor (de 500). ${ }^{30}$

Cabe mencionar que 10 a $20 \%$ de los pacientes con síndrome de Down desarrollan una llamada leucemia transitoria, también conocida como trastorno mieloproliferativo transitorio o mielopoyesis anormal transitoria. Ésta es una forma de leucemia casi exclusiva de los recién nacidos con síndrome de Down, la cual suele acompañarse de mutaciones en el gen del factor de transcripción hematopoyético GATA1 (Xp11.23) y aunque suele resolverse espontáneamente a los 3 meses de edad $20 \%$ de los pacientes recuperados de una leucemia transitoria desarrollan leucemia megacarioblástica en los primeros 4 años de vida y ésta siempre se acompaña de mutaciones somáticas en GATA1, lo cual indica que las mutaciones en este gen podrían ser un evento in utero y que los blastos de leucemia megacarioblástica podrían derivar de subclonas persistentes de células de leucemia transitoria como resultado de mutaciones adicionales. ${ }^{31}$ Además, estudios en humanos con distintas trisomías 21 parciales identificaron una región de $8.35 \mathrm{Mb}$ en Hsa21, que involucra a los genes RUNX1 (21q22.3), ERG (21q22.2) y ETS2 (21q22.3) como candidatos para el desarrollo de leucemia megacarioblástica en síndrome de Down. Particularmente, el factor de transcripción $R U N X 1$ está involucrado en la megacariopoyesis y mantenimiento de células troncales hematopoyéticas. ${ }^{12}$

\section{Tumores sólidos}

El riesgo para tumores sólidos es menor en pacientes con síndrome de Down. Ratones heterocigotos para mutaciones en el gen $A p c(A P C, 5 q 22.2)$ cruzados con modelos trisómicos para 33 genes con homología a genes humanos en el Hsa21, mostraron una clara reducción en la frecuencia de tumores en la descendencia trisómica comparada con la descendencia euploide. El gen Ets2 (ETS2, 21q22.3), incluido en esta región, inhibió el crecimiento de tumores cuando se expresaba en tres copias, a la inversa, cuando se reduce a una copia, resultó en incremento en la tasa de tumores. ${ }^{32}$

Recientemente se ha postulado que una disminución en la angiogénesis podría impedir el crecimiento de tumores en el síndrome de Down. En este contexto, DSCR1 ha mostrado inhibir la angiogénesis inducida por el factor de crecimiento vasculoendotelial. Otros genes que han disminuido la angiogénesis en modelos ratones incluyen a Adamts 1, Erg, Jam2 y Pttg 1 ip..33

\section{Cardiopatías congénitas}

Cerca de $50 \%$ de los pacientes con síndrome de Down tiene una cardiopatía congénita. ${ }^{8}$ En uno de los estudios poblacionales más grandes las malformaciones más frecuentes fueron canal atrioventricular completo, comunicación interventricular, comunicación interatrial, tetralogía de Fallot y persistencia del conducto arterioso. ${ }^{34}$ Datos en población mexicana indican una prevalencia de cardiopatías congénitas (en el síndrome de Down) de 58\%; sin embargo, en contraste con los informes a nivel mundial el canal atrioventricular completo muestra una frecuencia mucho menor que las comunicaciones interventricular e interatrial (Cuadro 2). ${ }^{35}$ En 2008, el National Down Syndrome Project of United States of America encontró que ser descendiente de hispanos confiere una oportunidad relativa de 0.48 para canal atrioventricular completo. ${ }^{36}$

Los mecanismos genómicos implicados en esta variabilidad siguen siendo ampliamente discutidos. Debido a su alta frecuencia, comparada con 
Cuadro 2. Prevalencia de cardiopatías congénitas reportadas a nivel mundial y en México

\begin{tabular}{|l|c|c|}
\hline Tipo de cardiopatía & \% mundial & \% México \\
\hline Canal atrioventricular & 37 & 8 \\
\hline Comunicación intervertricular & 31 & 22 \\
\hline Comunicación interauricular & 15 & 24 \\
\hline Tetralogía de Fallot & 5 & 0.6 \\
\hline $\begin{array}{l}\text { Persistencia de conducto } \\
\text { arterioso }\end{array}$ & 4 & 21 \\
\hline
\end{tabular}

Irving C et al. Arch Dis Child.2012 Apr;97(4):326-30; de Rubens J et al. Rev Esp Cardiol.2003 Sep.56(9):894-9.

individuos sin síndrome de Down y su espectro clínico variable, las cardiopatías congénitas se han utilizado como modelo en la búsqueda de loci de riesgo, mutaciones patogénicas, variación de dosis génica y variantes en número de copias (CNVs por sus siglas en ingles) que expliquen esta compleja etiopatogenia del fenotipo del síndrome de Down. ${ }^{37}$

Análisis en humanos con diferentes trisomías, 21 parciales, llevó a la identificación de una región de 1.77 Mb que contiene 10 genes. De estos, DSCAM (21q22.2) es el único que se expresa en el corazón en desarrollo, por lo que se planteó como un gen candidato; este análisis descarta genes previamente identificados como candidatos, entre ellos DIRK1A y COL6A1 (21q22.3), genes altamente expresados en las almohadillas endocárdicas a nivel atrioventricular en fetos con síndrome de Down. (12) Desafortunadamente, no se han encontrado defectos cardiacos en modelos ratones trisómicos para 33 genes, incluyendo DSCAM.

Fuera del Hsa21, mutaciones en CRELD1 (3p25.3) se han relacionado con canal atrioventricular completo en población general y se ha postulado su asociación con dicho defecto en pacientes con síndrome de Down. Otros estudios han mostrado que polimorfismos en los genes SLC19A1 (21q22.3) y MTHFR (1p36.3) involucrados en la vía del folato, están asociados con canal atrioventricular completo en pacientes con síndrome de Down. ${ }^{38}$

Dentro de las variantes en número de copias del cromosoma 21 asociadas con cardiopatía están algunas duplicaciones que involucran a regiones reguladoras del gen RIPK4 (21q22.2), el cual se ha asociado con morfogénesis epitelial (RR: 2.29) y del gen ZBTB21 que participa en la regulación de la vía de señalización de WNT/beta-catenina, vía requerida para la diferenciación cardiaca de células embrionarias (RR: 1.84). Además, se han identificado variantes en número de copias fuera del cromosoma 21, sobre todo regiones previamente asociadas con cardiopatías congénitas no sindromáticas. Este grupo afecta a genes del cilioma, el cual es un componente crítico de la septación atrioventricular ${ }^{39}$ (Cuadro 3).

Finalmente, es importante resaltar que a pesar de todos estos avances existen aún diversas manifestaciones clínicas frecuentes en paciente con síndrome de Down que aún no tienen una correlación con genes específicos, como alteraciones oftalmológicas, audiológicas, hipotiroidismo, alteraciones dermatológicas, genitourinarias como criptorquidia, hipospadias, malformaciones renales, así como la desregulación inmunológica que se asocia con infecciones recurrentes.

Cuadro 3. Variantes en número de copias raras asociadas con cardiopatía congénita en síndrome de Down

\begin{tabular}{|c|c|c|}
\hline Locus & $\begin{array}{l}\text { Variantes en número } \\
\text { de copias }\end{array}$ & $\begin{array}{c}\text { Genes } \\
\text { identificados }\end{array}$ \\
\hline $2 \mathrm{p} 21$ & Duplicación (584.5kb) & $\begin{array}{l}\text { LINC01121, } \\
\text { SRBD1, RKCE }\end{array}$ \\
\hline $2 q 12.3$ & Duplicación (784.7kb) & LIMS1 \\
\hline $2 q 13$ & Deleción (583.3-559.3kb) & MIR4267 \\
\hline $8 p 23.1$ & Deleción (79.1kb) & $X K R 5, D E F B 1$ \\
\hline $8 \mathrm{p} 23.1$ & $\begin{array}{l}\text { Duplicación } \\
(251-524.7 \mathrm{~kb})\end{array}$ & $\begin{array}{c}\text { CTSB, DEFB134, } \\
\text { DEFB136, } \\
\text { DEFB135 }\end{array}$ \\
\hline $15 q 13.3$ & Duplicación (514kb) & CHRNA7 \\
\hline $22 q 11.21$ & Deleción (957.8kb) & PPM1F, ТОР3В \\
\hline
\end{tabular}




\section{CONCLUSIONES}

El estudio de los genes implicados en el síndrome de Down continúa siendo un tema central, ofreciendo grandes retos para su análisis. La información obtenida hasta el momento no ha permitido aclarar con exactitud los genes sensibles a dosis implicados en el fenotipo de estos pacientes, lo cual podría explicarse por el efecto de múltiples genes implicados y la compleja interacción entre ellos. Por otro lado, muchas de las manifestaciones clínicas de los pacientes con síndrome de Down se comportan como entidades multifactoriales, donde no existe un solo gen causal involucrado, sino un grupo de genes que interactúan entre sí y con el ambiente, lo cual confiere una alta complejidad a la fisiopatología subyacente en esta enfermedad.

\section{REFERENCIAS}

1. Weijerman $\mathrm{M}$, de Winter J. Clinical practice. The care of children with Down syndrome. Eur J Pediatr. 2010 Dec;169(12):1445-52.

2. Ivan D, Cromwell P. Clinical Practice Guidelines for Management of Children With Down Syndrome: Part I. J Pediatr Health Care. 2014 Jan;28(1):105-10.

3. Down J. Observations on an ethnic classification of idiots. 1866. Ment Retard. 1995 Feb;33(1):54-6.

4. Lejeune J, Gautier M, Turpin R. Etude des chromosomes somatiques de neuf enfants mongoliens. C R Hebd Seances Acad Sci. 1959 Mar;248(11):1721-2.

5. Organización Mundial de la Salud [Internet]. Genomic resource centre. (2015). En http://www.who.int/genomics/en/

6. Secretaría de Salud. Atención integral de la persona con síndrome de Down. Lineamiento técnico 2007. Centro Nacional de Equidad de Género y Salud Reproductiva; 2007. En http://www.salud.gob.mx/unidades/cdi/documentos/ Sindrome_Down_lin_2007.pdf

7. International Clearinghouse for Birth Defects Surveillance and Research. Annual Report 2012. Roma Italy; The International Centre on Birth Defects-ICBDSR; 2012. Mexico:RYVEMCE. 163-8.

8. Hall B. Mongolism in newborn infants. An examination of the criteria for recognition and some speculations on the pathogenic activity of the chromosomal abnormality. Clin Pediatr.1966;5(1):4-12.

9. Freeman S, Bean L, Allen E, Tinker S, Locke A, Druschel C, et al. Ethnicity, sex, and the incidence of congenital heart defects: a report from the National Down Syndrome Project. Genetics in Medicine.2008;10(3):173-80.

10. Geelhoed E, Bebbington A, Bower C, Deshpande A, Leonard $H$. Direct health care costs of children and adolescents with Down syndrome. J Pediatr.2011 Oct;159(4):541-5.

11. Baraona F, Gurvitz M, Landzberg M, Opotowsky A. Hospitalizations and mortality in the United States for adults with Down syndrome and congenital heart disease. Am J Cardiol.2013 Apr;111(7):1046-51.

12. Korbel J, Tirosh-Wagner T, Urban A, Chen X, Kasowski M, Dai $L$, et al. The genetic architecture of Down syndrome phenotypes revealed by high-resolution analysis of human segmental trisomies. Proc Natl Acad Sci USA.2009 Jul;106(29): 12031-6.

13. Roizen N, Patterson D. Down's Syndrome. Lancet.2003 Jan;361(9365):1281-9.

14. Morice E, Andreae L, Cooke S, Vanes L, Fisher E, Tybulewicz $V$ et al. Preservation of long-term memory and synaptic plasticity despite short-term impairments in the Tc1 mouse model of Down syndrome. Learn Mem.2008 Jul;15(7):492-500.

15. Lyle R, Bena F, Gagos S, Gehrig C, Lopez G, Schinzel A et al. Genotype-phenotype correlations in Down syndrome identified by array CGH in 30 cases of partial trisomy and partial monosomy chromosome 21. Eur J Hum Genet.2009 Apr;17(4):454-66.

16. Duchon A, Pothion S, Brault V, Sharp A, Tybulewicz V, Fisher E et al. The telomeric part of the human chromosome 21 from Cstb to Prmt2 is not necessary for the locomotor and shortterm memory deficits observed in the Tc1 mouse model of Down syndrome. Behav Brain Res;2011 Mar;217(2):271-81.

17. Altafaj X, Dierssen M, Baamonde C, Marti E, Visa J, Guimera $J$ et al. Neurodevelopmental delay, motor abnormalities and cognitive deficits in transgenic mice overexpressing Dyrk1A (minibrain), a murine model of Down's syndrome. Hum Mol Genet.2001 Sep;10(18):1915-23.

18. Ema M, Ikegami S, Hosoya T, Mimura J, Ohtani H, Nakao $\mathrm{K}$ et al. Mild impairment of learning and memory in mice overexpressing the $\mathrm{mSim} 2$ gene located on chromosome 16, an animal model of Down's syndrome. Hum. Mol. Genet199 Aug;8(8):1409-15.

19. Alves-Sampaio A, Troca-Marin J, Montesinos M. NMDA mediated regulation of DSCAM dendritic local translation is lost in a mouse model of Down's syndrome. J Neurosci.2010 Oct;30(40):13537-48.

20. Best T, Siarey R, Galdzicki Z. Ts65Dn, a mouse model of Down syndrome, exhibits increased GABAB-induced potassium current..J Neurophysiol.2007 Jan;97:892-900.

21. Voronov S, Frere S, Giovedi S, Pollina E, Borel C, Zhang H, et al. Synaptojanin 1-linked phosphoinositide dyshomeostasis and cognitive deficits in mouse models of Down's syndrome. Proc Natl Acad Sci USA.2008 Jun;105(27):9415-20.

22. Netzer W, Powell C, Nong Y, Blundell J, Wong L, Duff $K$ et al. Lowering beta-amyloid levels rescues learning and me- 
mory in a Down syndrome mouse model. PLoS One.2010 Jun;5(6):1-5.

23. Olson L, Roper R, Baxter L, Carlson E, Epstein C, Reeves R. Down syndrome mouse models Ts65Dn, Ts1Cje, and Ms1Cje/Ts65Dn exhibit variable severity of cerebellar phenotypes. Dev Dyn.2004Jul;230(3):581-9.

24. Trazzi S, Mitrugno V, Valli E, Fuchs C, Rizzi S, Guidi et al. APPdependent up regulation of Ptch1 underlies proliferation impairment of neural precursors in Down syndrome. Hum Mol Genet.2011 Apr;20(8):1560-73.

25. Chang K, Min K. Upregulation of three Drosophila homologs of human chromosome 21 genes alters synaptic function: implications for Down syndrome. Proc Natl Acad Sci USA.2009 Sep;106(40):17117-22.

26. McNaughton D, Knight W, Guerreiro R, Ryan N, Lowe J, Poulter $\mathrm{M}$ et al. Duplication of amyloid precursor protein (APP), but not prion protein (PRNP) gene is a significant cause of early onset dementia in a large UK series. Neurobiol Aging.2012 Feb;33(2):426.e13-21.

27. Ryoo $S$, Cho $H$, Lee $H$, Jeong $H$, Radnaabazar $C$, Kim $Y$ et al.. Dual-specificity tyrosine(Y)-phosphorylation regulated kinase $1 \mathrm{~A}$-mediated phosphorylation of amyloid precursor protein: evidence for a functional link between Down syndrome and Alzheimer's disease. J Neurochem.2008 Mar;104(5):1333-44.

28. Richtsmeier J, Zumwalt A, Carlson E, Epstein C, Reeves R. Craniofacial phenotypes in segmentally trisomic mouse models for Down syndrome. Am J Med Genet.2002 Feb;107(4):317-24.

29. Sumarsono S, Wilson T, Tymms M, Venter D, Corrick C, Kola $\mathrm{R}$, et al. Down's syndrome-like skeletal abnormalities in Ets2 transgenic mice. Nature.1996 Feb;379:534-7.

30. Hasle, H., Clemmensen, I. H. and Mikkelsen, M. Risks of leukaemia and solid tumours in individuals with Down's syndrome. Lancet 355, 165-169.
31. Hitzler J, Zipursky A. Origins of leukaemia in children with Down syndrome. Nat Rev Cancer.2005 Jan; 5(1):11-20.

32. 32. Sussan T, Yang A., Li F, Ostrowski M, Reeves R. Trisomy represses $\mathrm{Apc}(\mathrm{Min})$-mediated tumours in mouse models of Down's syndrome. Nature.2008 Jan;451(7174):73-5.

33. 33. Reynolds, L. E., Watson, A. R., Baker, M., Jones, T. A., D’Amico, G., Robinson, S. D., Joffre, C., Garrido-Urbani, S., Rodriguez-Manzaneque, J. C., Martino-Echarri, E. et al.. Tumour angiogenesis is reduced in the Tc1 mouse model of Down's syndrome. Nature. (2010); 465: 813-817.

34. 34. Irving C, Chaudhari M. Cardiovascular abnormalities in Down's syndrome: spectrum, management and survival over 22 years. Arch Dis Child.2012 Apr;97(4):326-30.

35. 35. de Rubens J, del Pozzo B, Pablos J, Calderón C, Castrejón R. Heart Malformations in Children With Down Syndrome. Rev Esp Cardiol.2003 Sep.56(9):894-9

36. 36. Freeman $S$, Bean L, Allen E, Tinker S, Locke A, Druschel $C$ et al. Ethnicity, sex, and the incidence of congenital heart defects: a report from the National Down Syndrome Project. Geneti Med.2008 Mar;10(3):173-80.

37. 37. Sailani $M$, Makrythanasis $P$, Valsesia $A$, Santoni $F$ Deutsch S, Popadin $\mathrm{K}$ et al. The complex SNP and CNV genetic architecture of the increased risk of congenital heart defects in Down Syndrome. Genom Res.2013 Jun;23(9):1410-21.

38. 38. Liu C, Morishima M, Yu T, Matsui S, Zhang L, Fu D, et al. Genetic analysis of Down syndrome-associated heart defects in mice. Hum Genet.2011 Nov;130(5):623-32.

39. 39. Ramachandran D, Mulle J, Locke A, Bean L, Rosser T, Bose et al. Contribution of copy-number variation to Down syndrome-associated atrioventricular septal defects. Genet Med.2015 Jul;17(7):554-60. 